\title{
Dynamic Modelling and Vibration Control of a Planar Parallel Manipulator with Structurally Flexible Linkages
}

\author{
Bongsoo Kang ${ }^{1}$ and James K. Mills ${ }^{2}$ \\ Hannam University ${ }^{1}$, \\ University of Toronto ${ }^{2}$ \\ South Korea ${ }^{1}$ \\ Canada ${ }^{2}$
}

\section{Introduction}

A parallel manipulator provides an alternative design to serial manipulators, and can be found in many applications such as mining machines (Arai et al., 1991). Through the design of active joints such that actuators are fixed to the manipulator base, the mass of moving components of the parallel manipulator is greatly reduced, and high speed and high acceleration performance may be achieved. Parallel manipulators, comprised of closed-loop chains due to multiple linkages of the parallel structure, also provide high mechanical rigidity, but adversely exhibit smaller workspace and associated singularities. Considerable research has focused on kinematic analysis and singularity characterization of these devices (Gosselin \& Angeles, 1990, Merlet, 1996). Planar parallel manipulators typically consist of three closed chains and a moving platform. According to the arrangement of their joints in a chain, these mechanisms are classified as PRR, RRR etc. where P denotes a prismatic joint and $\mathrm{R}$ denotes a revolute joint respectively.

E An assembly industry, such as the electronic fabrication, demands high-speed, high o. acceleration placement manipulators, with corresponding lightweight linkages, hence these linkages deform under high inertia forces leading to unwanted vibrations. Moreover, such multiple flexible linkages of a parallel manipulator propagate their oscillatory motions to the moving platform where a working gripper is located. Therefore, such vibration must be damped quickly to reduce settling time of the manipulator platform position and orientation. A number of approaches to develop the dynamic model of parallel manipulators with structural flexibility have been presented in the literature(Fattah et al., 1995, Toyama et al. 2001), but relatively few works related to vibration reduction of a parallel manipulator have been published. Kozak (Kozak et al., 2004) linearized the dynamic equations of a two-degree-of-freedom parallel manipulator locally, and applied an input is shaping technique to reduce residual vibrations through modification of the reference command given to the system. Kang (Kang et al., 2002) modeled a planar parallel manipulator using the assumed modes method, and presented a two-time scale controller Ð for linkage vibration attenuation of the planar parallel manipulator. Since both the input shaping technique and the two-time scale control scheme, applied to parallel manipulators, 
can only control command inputs to joint actuators of a manipulator, their performance on vibration reduction of flexible linkage are limited.

This chapter introduces a methodology for the dynamic analysis of a planar parallel mechanism beginning with the rigid-body model of a planar parallel manipulator. Flexible deformations of each linkage are expressed by the product of time-dependant functions and position-dependant functions, i.e. an assumed modes model. Overall dynamic equations of the motion for a planar parallel manipulator are formulated by Lagrangian equations. Then, an active damping approach using piezoelectric material actuators is presented to damp out oscillation of linkages of a planar parallel manipulator. Also an integrated control scheme is designed to permit the platform of the parallel manipulator to follow a given trajectory while simultaneously damping structural vibration of flexible linkages. Attached directly to the surface of flexible linkages, piezoelectric materials deform under an applied control voltage, producing shear forces, which counteract shear stresses that occur due to deformation of the linkages. Transducers are often developed from either of two types of material: polyvinylidene fluoride (PVDF) or lead zirconium titanate (PZT). PVDF is lightweight and is mainly used as a measurement device for detecting vibration, although the PVDF can also be used as an actuator. PZT has been used as actuators for micro mechanisms and has a higher strain constant than PVDF.

In simulations, both the PZT and PVDF piezoelectric actuators are applied to a planar parallel manipulator with flexible linkages and the respective performance of two actuators are compared. The dynamics of the planar parallel platform are selected such that the linkages have considerable flexibility, to better exhibit the effects of the vibration damping control system proposed. Simulation results show that the PZT actuator can give better performance in vibration attenuation than the PVDF layer.

\section{Rigid-body analysis of a planar parallel manipulator}

\subsection{Architecture of a planar parallel manipulator}

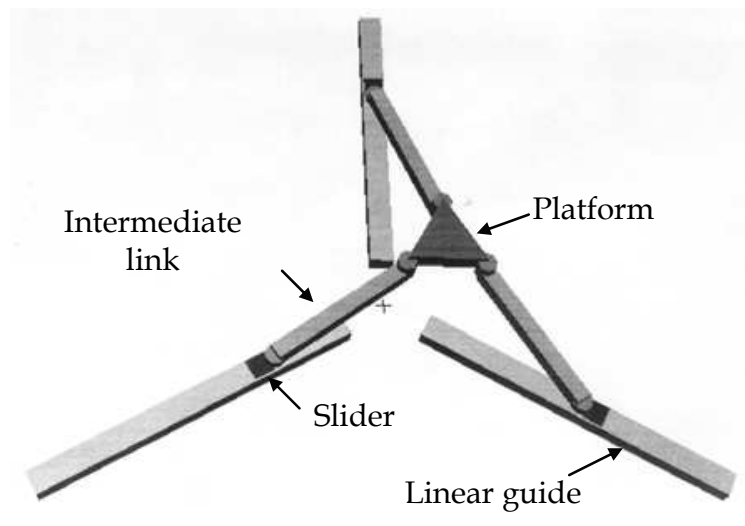

Fig. 1. Configuration of a $\underline{P R R}$ type planar parallel manipulator

The architecture of a PRR type parallel mechanism is illustrated in Fig. 1. The underline of the first character of $\underline{P} R R$ means that the first Prismatic joint is an active joint derived by 
actuators. The moving platform, a regular triangular shape, exhibits translation and rotational motion in a plane. Three intermediate links between the moving platform and sliders play a role to convert the actuating force into movements of the platform. Both ends of the intermediate link are composed of non-actuated revolute joints. The sliders move along the linear guide and their motions can be achieved by a ball-screw mechanism. The proposed planar manipulator is categorized as a PRR type, because a closed-loop chain consists of a prismatic joint and two consecutive revolute joints. In contrast to well-known RPR type parallel manipulators, actuators of the proposed PRR configuration remain stationary that results in low inertia of moving parts. Workspace analysis of a planar parallel manipulator has been addressed (Gosselin et al., 1996, Heerah et al., 2002) and singularity analysis of a planar parallel manipulator has been studied (Gosselin \& Angeles, 1990, Merlet, 1996).

\subsection{Kinematics}

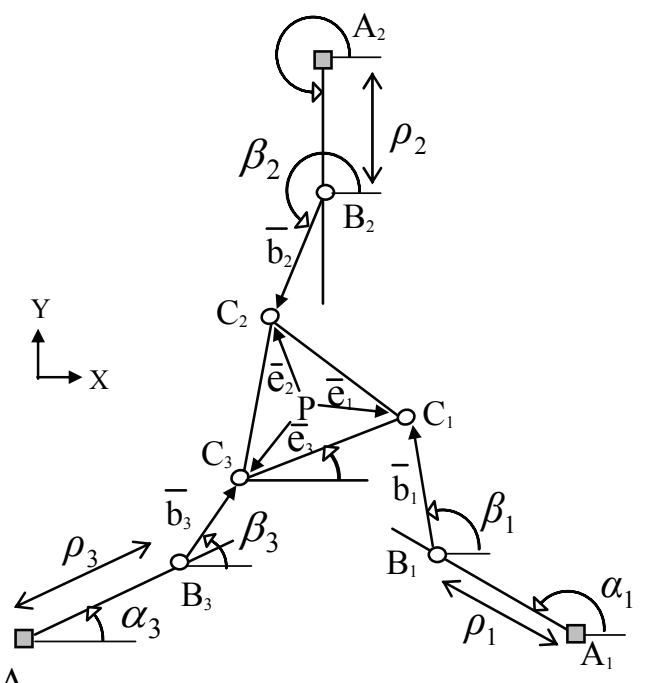

$\mathrm{A}_{3}$

Fig. 2. Coordinate system of rigid-body model

We will begin with formulation of the rigid-body model of the proposed planar parallel manipulator, and then include structural flexibility of the linkage in Section 3. Prior to derivation of dynamics of the parallel manipulator, the inverse kinematic solution of the manipulator is formulated to define kinematic relations between active joints, and position and orientation of the platform. Then, based on these kinematic relations, equations of the motion for a parallel manipulator are formulated later.

Generalized coordinates for the PRR manipulator are defined, as shown in Fig. 2. The position of the reference X-Y frame is arbitrary. $A_{i}$ is the origin of the $i^{\text {th }}$ linear guide and $B_{i}$ is the position of the $i^{\text {th }}$ slider. $C_{i}$ is the position of the revolute joint of the platform facing with the $i^{\text {th }}$ linkage and $P$ is the position of the platform at its mass center. Three linkages, including associated coordinates, are numbered with a subscript, $i$, starting from the lower 
right link in a counterclockwise direction. The pose of the moving platform at its mass center can be written with respect to the reference $\mathrm{X}-\mathrm{Y}$ frame as

$$
\bar{X}_{P}:=\left[\begin{array}{lll}
x_{P} & y_{P} & \phi
\end{array}\right]^{T}
$$

The displacement of the sliders from their origin, $A_{i}$, to $B_{i}$, are expressed as

$$
\bar{\rho}:=\left[\begin{array}{lll}
\rho_{1} & \rho_{2} & \rho_{3}
\end{array}\right]^{T}
$$

$\beta_{\mathrm{i}}$ is defined as the angle at $B_{i}$ between the $\mathrm{X}$-axis of the fixed frame and the $i^{\text {th }}$ intermediate link and $\alpha_{i}$ is the angle at $A_{i}$ between the $\mathrm{X}$-axis of the fixed frame and the $i^{\text {th }}$ linear guide. The position and orientation of the $i^{\text {th }}$ link at its mass center can also be written as

$$
\bar{X}_{i}:=\left[\begin{array}{lll}
x_{i} & y_{i} & \beta_{i}
\end{array}\right]^{T}
$$

For each chain, a loop close equation can be written using position vectors defined as

$$
\bar{A}_{i} \bar{P}+\bar{P} \bar{C}_{i}=\bar{A}_{i} \bar{B}_{i}+\bar{B}_{i} \bar{C}_{i} \quad i=1,2,3
$$

Fig. 3. shows the diagram of the loop close equation when the first link is considered. The right-hand side of equation (4), the coordinates of $C_{i}$, is written as

$$
\begin{gathered}
x_{c i}=x_{a i}+\rho_{i} \cos \alpha_{i}+l \cos \beta_{i} \\
y_{c i}=y_{a i}+\rho_{i} \sin \alpha_{i}+l \sin \beta_{i}
\end{gathered}
$$

where $x_{a i}$ and $y_{a i}$ are coordinates of point $A_{i}$ respectively and $l$ is length of the linkage.

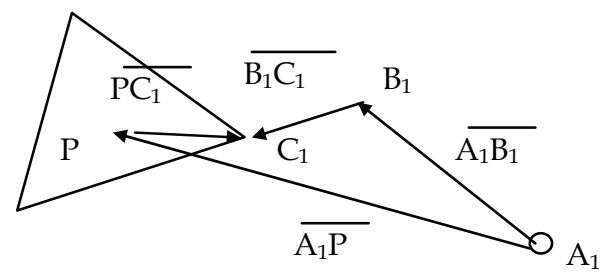

Fig. 3. Schematic diagram of loop close equation

Also, the $x$ and $y$ coordinates of point $C_{i}$, i.e., the left-hand side of equations (4), can be formulated using platform coordinates as

$$
\begin{aligned}
& x_{c i}=x_{p}+x_{c i}^{\prime} \cos \phi-y_{c i}^{\prime} \sin \phi \\
& y_{c i}=y_{p}+x_{c i}^{\prime} \sin \phi+y_{c i}^{\prime} \cos \phi
\end{aligned}
$$

$x_{c i}^{\prime}$ and $y_{c i}^{\prime}$ are $\mathrm{x}$ and $\mathrm{y}$ coordinates of $\mathrm{Ci}$ respectively measured from the mass center of the platform, $P$, when $\phi$ is zero, as shown in Fig. 4. 


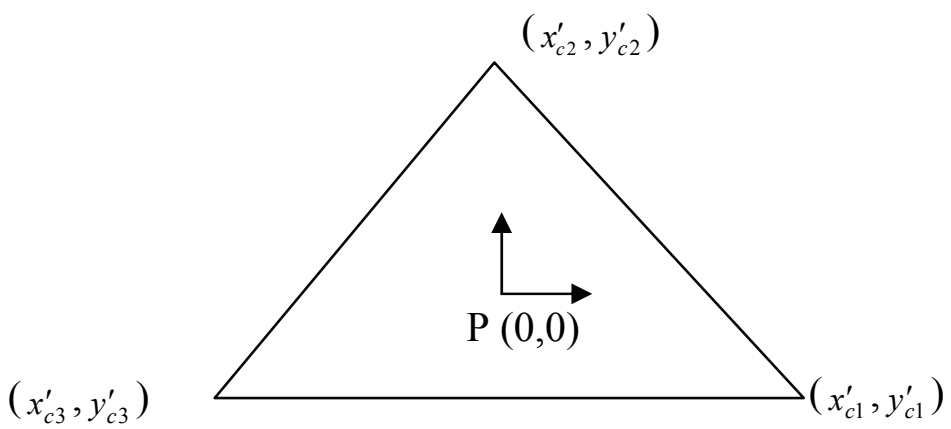

Fig. 4. Platform coordinates

From equations (5-8), a closed-form solution is calculated as

$$
\rho_{i}=M_{i} \pm \sqrt{l^{2}-S_{i}^{2}} \quad i=1,2,3
$$

where:

$$
\begin{gathered}
M_{i}=\left(x_{c i}-x_{a i}\right) \cos \alpha_{i}+\left(y_{c i}-y_{a i}\right) \sin \alpha_{i} \\
S_{i}=\left(x_{c i}-x_{a i}\right) \sin \alpha_{i}-\left(y_{c i}-y_{a i}\right) \cos \alpha_{i}
\end{gathered}
$$

Since there are two possible solutions for each chain, this manipulator can take on a maximum of eight configurations for a set of given coordinates of the platform. Note, only if the argument of the square root in equation (9) becomes zero, dose equation (9) have a unique solution. If the argument turns out to be negative, there is no solution to satisfy given kinematic requirements. Compared with the inverse kinematic solution described above, forward kinematic solutions of a planar parallel manipulator are much more difficult to solve (Merlet, 1996).

\section{Dynamic analysis of flexible linkages}

As industry demands high-speed machines, and hence lightweight linkages which deform under high inertial forces, we must consider structural flexibility of linkages in modeling a parallel manipulator. A single flexible link has been modeled in (Bellezza et all., 1990) and a serial type manipulator with both rigid and flexible links has been presented in (Low \& Vidyasagar, 1988). Flexible models of a parallel manipulator have been studied in (Fattah et al., 1995).

A coordinate system for the flexible model is identical with the rigid-body model shown in Fig. 2. Only difference is the existence of the lateral deformation, $w_{i}(l)$, at the distal end of the $i^{\text {th }}$ linkage, $C_{i}$, due to flexibility of the linkage, as shown in Fig. 5. Out of X-Y plane deformations are not considered here. This is the subject of another analysis. If length of the linkage, $l$, is much longer than thickness of the linkage, the linkage can be treated as an Euler-Bernoulli beam (Genta, 1993). 


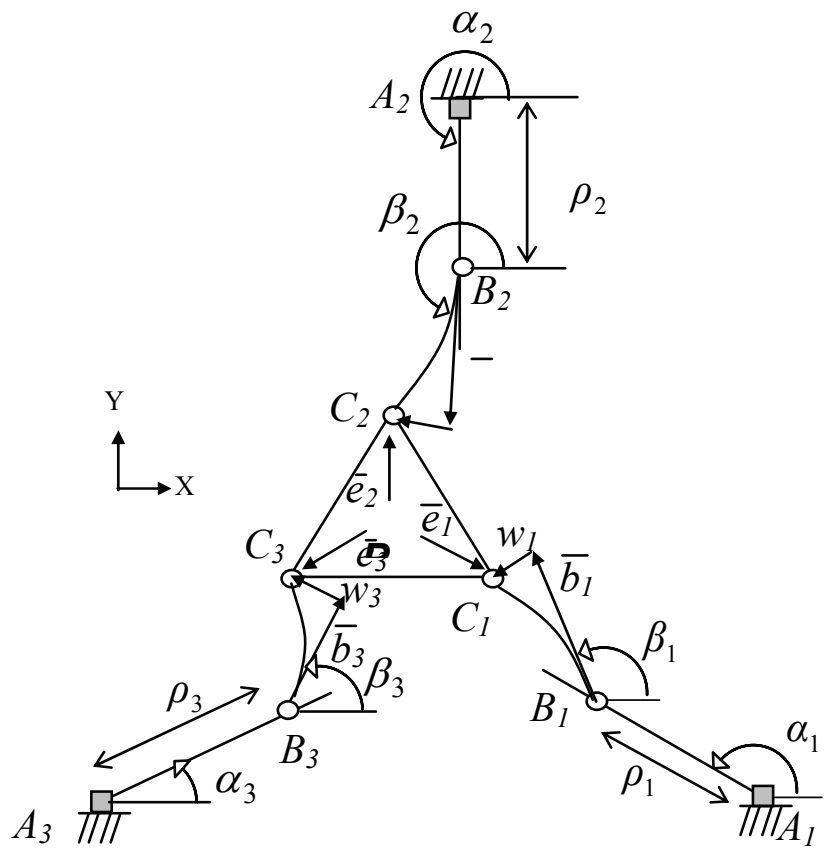

Fig. 5. Coordinate system of structurally-flexible model

Coupled with flexible deformations, the kinematic equations (5-6) are hence modified as follows;

$$
\begin{aligned}
& x_{c i}=x_{a i}+\rho_{i} \cos \alpha_{i}+l \cos \beta_{i}-w_{i}(l) \sin \beta_{i} \\
& y_{c i}=y_{a i}+\rho_{i} \sin \alpha_{i}+l \sin \beta_{i}+w_{i}(l) \cos \beta_{i}
\end{aligned}
$$

Since the left-hand side of equation (4) remains valid for a flexible model,

$$
\begin{aligned}
& x_{c i}=x_{p}+x_{c i}^{\prime} \cos \phi-y_{c i}^{\prime} \sin \phi \\
& y_{c i}=y_{p}+x_{c i}^{\prime} \sin \phi+y_{c i}^{\prime} \cos \phi
\end{aligned}
$$

Through Equations (10-13), the inverse kinematic solution of a structurally flexible manipulator is formulated as

$$
\rho_{i}=M_{i} \pm \sqrt{l^{2}+w_{i}(l)^{2}-S_{i}^{2}} \quad i=1,2,3
$$

where:

$$
M_{i}=\left(x_{c i}-x_{a i}\right) \cos \alpha_{i}+\left(y_{c i}-y_{a i}\right) \sin \alpha_{i}
$$




$$
S_{i}=\left(x_{c i}-x_{a i}\right) \sin \alpha_{i}-\left(y_{c i}-y_{a i}\right) \cos \alpha_{i}
$$

Comparing with the inverse kinematic solution of the rigid-body model, equation(9), the linkage deformation is added in the right-hand side of equation (14). In addition, large linkage deformation may lead to no solution to $\rho_{i}, i=1,2,3$, because the argument of the square root in equation (14) has a negative value.

Evaluation of the derivative of equations (10-13), with respect to time, gives

$$
\left(\dot{x}_{P} \bar{i}+\dot{y}_{P} \bar{j}\right)+\dot{\phi}\left(\bar{k} \times \bar{e}_{i}\right)=\dot{\rho}_{i} \bar{a}_{i}+\left(\dot{\beta}_{i}+\dot{w}_{i}(l) / l\right)\left(\bar{k} \times \bar{b}_{i}\right)
$$

where $\bar{i}, \bar{j}$ are unit vectors along the reference $\mathrm{X}-\mathrm{Y}$ frame respectively and $\bar{e}_{i}$ is shown in Fig. 5. Dot-multiplication of equation (15) by $\bar{b}_{i}$ leads to

$$
\dot{\rho}_{i}=\frac{1}{\bar{a}_{i} \cdot \bar{b}_{i}}\left[\begin{array}{lll}
b_{i x} & b_{i y} & e_{i x} b_{i y}-e_{i y} b_{i x}
\end{array}\right]\left[\begin{array}{lll}
\dot{x}_{P} & \dot{y}_{P} & \dot{\phi}
\end{array}\right]^{T}:=J_{P i} \dot{\bar{X}}_{P}
$$

Subscripts of a position vector, named as $\mathrm{x}$ and $\mathrm{y}$, represent $\mathrm{X}$-directional and Y-directional components of the corresponding vector respectively. Cross-multiplication of equation (15) by $\bar{b}_{i}$ gives

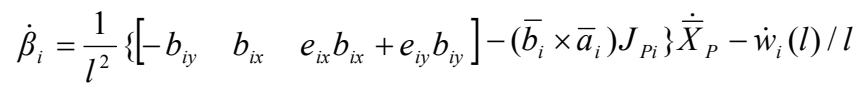

Accelerations of the sliders and the links are given respectively by

$$
\begin{array}{r}
\ddot{\rho}_{i}=\frac{1}{\bar{a}_{i} \cdot \bar{b}_{i}}\left\{\left[\begin{array}{lll}
b_{i x} & b_{i y} & e_{i x} b_{i y}-e_{i y} b_{i x}
\end{array}\right] \ddot{\bar{X}}_{P}-\bar{b}_{i} \cdot \bar{e}_{i} \dot{\phi}^{2}+\dot{\beta}_{i}^{2} l^{2}+\dot{\beta}_{i} \dot{w}_{i}(l) l\right\} \\
\ddot{\beta}_{i}=\frac{1}{l^{2}}\left\{\left[\begin{array}{lll}
-b_{i y} & b_{i x} & e_{i x} b_{i x}+e_{i y} b_{i y}
\end{array}\right] \ddot{\bar{X}}_{P}-\left(\bar{b}_{i} \times \bar{e}_{i}\right) \dot{\phi}^{2}-\left(\bar{b}_{i} \times \bar{a}_{i}\right) \ddot{\rho}_{i}\right\}-\ddot{w}_{i}(l) / l
\end{array}
$$

Since three linkages in this analysis are assumed to have structural flexibility, the linkage deforms under high acceleration, as shown in Fig. 5. Flexible deformations can be expressed by the product of time-dependant functions and position-dependant functions, i.e. an assumed modes model (Genta, 1993);

$$
w_{i}(x, t):=\sum_{j=1}^{r} \eta(t)_{i j} \psi_{j}(\xi) \quad i=1,2,3
$$

where:

$\xi:=x / l, r:=$ the number of assumed modes.

Functions $\eta(\mathrm{t})$ can be considered the generalized coordinates expressing the deformation of the linkage and functions $\psi(\xi)$ are referred to as assumed modes.

Considering boundary conditions of the linkage on $B_{i}$ and $C_{i}$, their behavior is close to a pin $\left(B_{i}\right)$-free $\left(C_{i}\right)$ motion. Normalized shape functions, satisfying this boundary condition, are selected as: 


$$
\psi_{j}(\xi):=\frac{1}{2 \sin \left(\gamma_{j}\right)}\left[\sin \left(\gamma_{j} \xi\right)+\frac{\sin \left(\gamma_{j}\right)}{\sinh \left(\gamma_{j}\right)} \sinh \left(\gamma_{j} \xi\right)\right]
$$

where:

$$
0 \leq \xi \leq 1 \text { and } \gamma_{j}:=(j+0.25) \pi l \quad j=1,2, \ldots, r
$$

The first four shape functions are shown in Fig. 6 where the left end $\left(B_{i}\right)$ exhibits zero deformation and the right end $\left(C_{i}\right)$ exhibits a maximum deformation, as expected. All generalized coordinates are collected to form of a single vector $X$ defined as:

$$
X:=\left[\begin{array}{llll}
\bar{\rho} & \bar{\beta} & \bar{X}_{P} & \bar{\eta}
\end{array}\right]^{T} \in R^{9+3 r}
$$

where:

$$
\begin{aligned}
\bar{\beta} & :=\left[\begin{array}{lll}
\beta_{1} & \beta_{2} & \beta_{3}
\end{array}\right]^{T} \\
\bar{\eta} & :=\left[\begin{array}{lllllllll}
\eta_{11} & \cdots & \eta_{1 r} & \eta_{21} & \cdots & \eta_{2 r} & \eta_{31} & \cdots & \eta_{3 r}
\end{array}\right]^{T}
\end{aligned}
$$

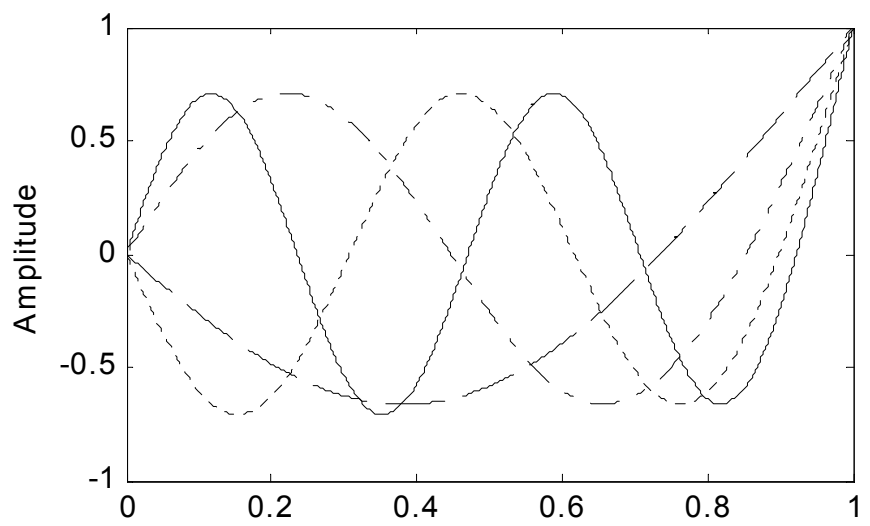

Fig. 6. Amplitude of first four mode shapes of straight beam vs. location along beam $\xi$ (dashed line: first mode, dash-dot line: second mode, dotted line: third mode, solid line: fourth mode)

Using inertia parameters of the manipulator and generalized coordinates, the kinetic energy of three sliders is written as

$$
T_{S}=\sum_{i=1}^{3} \frac{1}{2} m_{s} \dot{\rho}_{i}^{2}
$$

$m_{\mathrm{s}}$ is mass of the sliders.

The kinetic energy of the three links is expressed as

$$
T_{L}=\sum_{i=1}^{3} \frac{1}{2} \int \rho_{A}\left[\dot{\rho}_{i}^{2}+\left(x \dot{\beta}_{i}+\dot{w}_{i}\right)^{2}+2 \dot{\rho}_{i}\left(x \dot{\beta}_{i}+\dot{w}_{i}\right) \sin \left(\alpha_{i}-\beta_{i}\right)\right] d x
$$

The kinetic energy of the platform is expressed as 


$$
T_{P}=\frac{1}{2} m_{P}\left(\dot{x}_{P}^{2}+\dot{y}_{P}^{2}\right)+\frac{1}{2} I_{P} \dot{\phi}^{2}
$$

$m_{\mathrm{p}}, \mathrm{I}_{\mathrm{p}}$ are mass and mass moment of the platform respectively.

Therefore, collecting all kinetic energies, equations (23-25), the total kinetic energy of the system is

$$
\begin{gathered}
T=\sum_{i=1}^{3} \frac{1}{2} m_{s} \dot{\rho}_{i}{ }^{2}+\sum_{i=1}^{3} \frac{1}{2} \int \rho_{A}\left[\rho_{i}{ }^{2}+\left(x \dot{\beta}_{i}+\dot{w}_{i}\right)^{2}+2 \dot{\rho}_{i}\left(x \dot{\beta}_{i}+\dot{w}_{i}\right) \sin \left(\alpha_{i}-\beta_{i}\right)\right] d x \\
+\frac{1}{2} m_{P}\left(\dot{x}_{P}{ }^{2}+\dot{y}_{P}{ }^{2}\right)+\frac{1}{2} I_{P} \dot{\phi}^{2}
\end{gathered}
$$

Since gravitational force is applied along Z-direction, perpendicular to the $\mathrm{X}-\mathrm{Y}$ plane, potential energy due to gravitational force does not changed at all during any in-plane motions of the manipulator. Considering potential energy due to deformation of the linkage, total potential energy of the system is given as

$$
V=\sum_{i=1}^{3} \int E I\left(w_{i}^{\prime \prime}\right)^{2} d x
$$

where: $\rho_{A}:=$ mass per length of the linkage

$E \quad:=$ elastic modulus of the linkage

$I \quad:=$ area moment of inertia of the linkage

Evaluating Lagrangian equations of the first type given by

$$
\frac{d}{d t}\left(\frac{\partial T}{\partial \dot{X}_{i}}\right)-\frac{\partial(T-V)}{\partial X_{i}}=Q_{i}+\sum_{k=1}^{m} \lambda_{k} \frac{\partial \Gamma_{k}}{\partial X_{i}}, i=1,2, \ldots, 9+3 r
$$

where: $Q_{i}:=$ generalized force

$\lambda_{k}:=\quad k^{\text {th }}$ Lagrange multiplier

$\Gamma_{k}:=\quad k^{\text {th }}$ constrained equation

the left-hand side of equation (28) is formulated as follows:

$$
\begin{gathered}
\frac{d}{d t}\left(\frac{\partial T}{\partial \dot{\rho}_{i}}\right)-\frac{\partial(T-V)}{\partial \rho_{i}}=\left(m_{s}+m\right) \ddot{\rho}_{i}+0.5 m l \sin \left(\alpha_{i}-\beta_{i}\right) \ddot{\beta}_{i}+\sum_{j=1}^{r} \ddot{\eta}_{i j} \sin \left(\alpha_{i}-\beta_{i}\right) \int \rho_{A} \psi_{j} d x \\
-0.5 m l \cos \left(\alpha_{i}-\beta_{i}\right) \dot{\beta}_{i}^{2}-\sum_{j=1}^{r} \dot{\eta}_{i j} \dot{\beta}_{i} \cos \left(\alpha_{i}-\beta_{i}\right) \int \rho_{A} \psi_{j} d x \quad i=1,2,3 \\
\frac{d}{d t}\left(\frac{\partial T}{\partial \dot{\beta}_{i}}\right)-\frac{\partial(T-V)}{\partial \beta_{i}}=0.5 m l \sin \left(\alpha_{i}-\beta_{i}\right) \ddot{\rho}_{i}+m l^{2} \ddot{\beta}_{i} / 3+\sum_{j=1}^{r} \ddot{\eta}_{i j} \int \rho_{A} x \psi_{j} d x \\
+\sum_{j=1}^{r} \dot{\eta}_{i j} \dot{\rho}_{i} \cos \left(\alpha_{i}-\beta_{i}\right) \int \rho_{A} \psi_{j} d x \quad i=1,2,3
\end{gathered}
$$




$$
\begin{gathered}
\frac{d}{d t}\left(\frac{\partial T}{\partial \dot{X}_{P}}\right)-\frac{\partial(T-V)}{\partial X_{P}}=\left[\begin{array}{ccc}
m_{P} & 0 & 0 \\
0 & m_{P} & 0 \\
0 & 0 & I_{P}
\end{array}\right]\left[\begin{array}{c}
\ddot{x}_{P} \\
\ddot{y}_{P} \\
\ddot{\phi}
\end{array}\right] \\
\frac{d}{d t}\left(\frac{\partial T}{\partial \dot{\eta}_{i j}}\right)-\frac{\partial(T-V)}{\partial \eta_{i j}}=\sin \left(\alpha_{i}-\beta_{i}\right) \ddot{\rho}_{i} \int \rho_{A} \psi_{j} d x+\ddot{\beta}_{i} \int \rho_{A} x \psi_{j} d x+\ddot{\eta}_{i j} \int \rho_{A} \psi_{j}^{2} d x \\
-\int \rho_{A} \psi_{j} d x \cos \left(\alpha_{i}-\beta_{i}\right) \dot{\beta}_{i} \dot{\rho}_{i}+\int E I\left(\psi_{j}^{\prime \prime}\right)^{2} d x \quad i=1,2,3 \text { and } j=1,2, . ., r
\end{gathered}
$$

$m$ is mass of the linkage.

Since the number of generalized coordinates excluding vibration modes is nine, greater than the number of the degrees-of-freedom of the manipulator, three, six constraint equations should be considered in equations of the motion. From the geometry of three closed-loop chains, equation (4), a fundamental constrained equation is given by

$$
\bar{A}_{i} \bar{P}+\bar{P} \bar{C}_{i}-\bar{A}_{i} \bar{B}_{i}-\bar{B}_{i} \bar{C}_{i}=0 \quad i=1,2,3
$$

Dividing equations (33) into an X-axis's component and a Y-axis's component, six constraint equations are given by

$$
\begin{aligned}
& \Gamma_{2 i-1}:=\rho_{i} \cos \alpha_{i}+l \cos \beta_{i}-\sum_{j=1}^{r} \eta_{i j} \sin \beta_{i}-x_{P}-r \cos \left(\phi_{i}+\phi\right)=0 \\
& \Gamma_{2 i}:=\rho_{i} \sin \alpha_{i}+l \sin \beta_{i}+\sum_{i=1}^{r} \eta_{i j} \cos \beta_{i}-y_{P}-r \sin \left(\phi_{i}+\phi\right)=0
\end{aligned}
$$

where:

$$
r \cos \left(\phi_{i}\right):=x_{c i}^{\prime}, r \sin \left(\phi_{i}\right):=y_{c i}^{\prime} \quad i=1,2,3
$$

From equation (34) and (35), the right-hand side of equation (28) is

$$
\begin{gathered}
F_{a i}+\sum_{k=1}^{6} \lambda_{k} \frac{\partial \Gamma_{k}}{\partial \rho_{i}}=F_{a i}+\lambda_{2 i-1} \cos \alpha_{i}+\lambda_{2 i} \sin \alpha_{i} i=1,2,3 \\
\sum_{k=1}^{6} \lambda_{k} \frac{\partial \Gamma_{k}}{\partial \beta_{i}}=\lambda_{2 i-1}\left(-l \sin \beta_{i}-\sum_{j=1}^{r} \eta_{i j} \cos \beta_{i}\right)+\lambda_{2 i}\left(l \cos \beta_{i}-\sum_{j=1}^{r} \eta_{i j} \sin \beta_{i}\right) \\
F_{\text {ext }}+\sum_{k=1}^{6} \lambda_{k} \frac{\partial \Gamma_{k}}{\partial X_{P}}=F_{\text {ext }}+\left[\begin{array}{cccccc}
-1 & 0 & -1 & 0 & -1 & 0 \\
0 & -1 & 0 & -1 & 0 & -1 \\
s 3_{1} & c 3_{1} & s 3_{2} & c 3_{2} & s 3_{3} & c 3_{3}
\end{array}\right]\left[\begin{array}{c}
\lambda_{1} \\
\vdots \\
\lambda_{6}
\end{array}\right]
\end{gathered}
$$

where:

$s 3_{i}:=e_{i x}, c 3_{i}:=e_{i y}$

$F_{e x t}$ is an external force and $\mathrm{F}_{a i}$ is an actuating force. 


$$
\begin{aligned}
& \sum_{k=1}^{6} \lambda_{k} \frac{\partial \Gamma_{k}}{\partial \eta_{1 j}}=-\lambda_{1} \sin \beta_{1}+\lambda_{2} \cos \beta_{1} \quad j=1,2, . ., r \\
& \sum_{k=1}^{6} \lambda_{k} \frac{\partial \Gamma_{k}}{\partial \eta_{2 j}}=-\lambda_{3} \sin \beta_{2}+\lambda_{4} \cos \beta_{2} \quad j=1,2, . ., r \\
& \sum_{k=1}^{6} \lambda_{k} \frac{\partial \Gamma_{k}}{\partial \eta_{3 j}}=-\lambda_{5} \sin \beta_{3}+\lambda_{6} \cos \beta_{3}{ }^{j}=1,2, . ., r
\end{aligned}
$$

Putting equations (29-32) and equations (36-41) together, the equations of motion for the planar parallel manipulator are complete with a total of $9+3 \times r$ equations;

$$
\left[\begin{array}{cccc}
M_{11} & M_{12} & 0 & M_{14} \\
M_{12}^{T} & M_{22} & 0 & M_{24} \\
0 & 0 & M_{33} & 0 \\
M_{14}^{T} & M_{24}^{T} & 0 & M_{44}
\end{array}\right]\left[\begin{array}{c}
\ddot{\bar{\rho}} \\
\ddot{\bar{\beta}} \\
\ddot{\bar{X}}_{P} \\
\ddot{\bar{\eta}}
\end{array}\right]+\left[\begin{array}{c}
V_{1} \\
V_{2} \\
0 \\
V_{4}
\end{array}\right]+\left[\begin{array}{cccc}
0 & 0 & 0 & 0 \\
0 & 0 & 0 & 0 \\
0 & 0 & 0 & 0 \\
0 & 0 & 0 & K
\end{array}\right]\left[\begin{array}{c}
\bar{\rho} \\
\bar{\beta} \\
\bar{X}_{P} \\
\bar{\eta}
\end{array}\right]=\left[\begin{array}{c}
F_{a} \\
0 \\
F_{e x t} \\
0
\end{array}\right]+\left[\begin{array}{c}
J_{\Gamma 1} \\
J_{\Gamma 2} \\
J_{\Gamma 3} \\
J_{\Gamma 4}
\end{array}\right]\left[\begin{array}{c}
\lambda_{1} \\
\lambda_{2} \\
\lambda_{3} \\
\lambda_{4} \\
\lambda_{5} \\
\lambda_{6}
\end{array}\right]
$$

where:

$$
\begin{aligned}
& M_{11}=\left(m_{s}+m\right)\left[\begin{array}{ccc}
1 & 0 & 0 \\
0 & 1 & 0 \\
0 & 0 & 1
\end{array}\right] \in R^{3 \times 3} \quad M_{12}=\frac{m l}{2}\left[\begin{array}{ccc}
s_{1} & 0 & 0 \\
0 & s_{2} & 0 \\
0 & 0 & s_{3}
\end{array}\right] \in R^{3 \times 3} \\
& M_{14}=m\left[\begin{array}{ccccccccc}
s_{1} \int \psi_{1} d \xi & \cdots & s_{1} \int \psi_{r} d \xi & 0 & \cdots & 0 & 0 & \cdots & 0 \\
0 & \cdots & 0 & s_{2} \int \psi_{1} d \xi & \cdots & s_{2} \int \psi_{r} d \xi & 0 & \cdots & 0 \\
0 & \cdots & 0 & 0 & \cdots & 0 & s_{3} \int \psi_{1} d \xi & \cdots & s_{3} \int \psi_{r} d \xi
\end{array}\right] \in R^{3 \times 3 r} \\
& M_{22}=\frac{m l^{2}}{3}\left[\begin{array}{lll}
1 & 0 & 0 \\
0 & 1 & 0 \\
0 & 0 & 1
\end{array}\right] \in R^{3 \times 3} \quad M_{33}=\left[\begin{array}{ccc}
m_{p} & 0 & 0 \\
0 & m_{p} & 0 \\
0 & 0 & I_{P}
\end{array}\right] \in R^{3 \times 3} \\
& M_{24}=m l\left[\begin{array}{ccccccccc}
\int \psi_{1} \xi d \xi & \cdots & \int \psi_{r} \xi d \xi & 0 & \cdots & 0 & 0 & \cdots & 0 \\
0 & \cdots & 0 & \int \psi_{1} \xi d \xi & \cdots & \int \psi_{r} \xi d \xi & 0 & \cdots & 0 \\
0 & \cdots & 0 & 0 & \cdots & 0 & \int \psi_{1} \xi d \xi & \cdots & \int \psi_{r} \xi d \xi
\end{array}\right] \in R^{3 \times 3 r}
\end{aligned}
$$




$$
\begin{aligned}
& M_{44}=m\left[\begin{array}{ccc}
\hat{M} & 0 & 0 \\
0 & \hat{M} & 0 \\
0 & 0 & \hat{M}
\end{array}\right] \in R^{3 r \times 3 r} \quad \hat{M}=\left[\begin{array}{ccc}
\int \psi_{1}^{2} d \xi & \cdots & 0 \\
\vdots & \cdots & \vdots \\
0 & \cdots & \int \psi_{r}^{2} d \xi
\end{array}\right] \in R^{r \times r} \\
& K=\frac{E I}{l^{3}}\left[\begin{array}{ccc}
\hat{K} & 0 & 0 \\
0 & \hat{K} & 0 \\
0 & 0 & \hat{K}
\end{array}\right] \in R^{3 r \times 3 r} \hat{K}=\left[\begin{array}{ccc}
\int \psi_{1}^{\prime \prime 2} d \xi & \cdots & 0 \\
\vdots & \cdots & \vdots \\
0 & \cdots & \int \psi_{r}^{\prime \prime 2} d \xi
\end{array}\right] \in R^{r \times r} \\
& V_{1}=-\left[\begin{array}{c}
0.5 m l c_{1} \dot{\beta}_{1}^{2}+\sum_{j=1}^{r} m \dot{\eta}_{1 j} \dot{\beta}_{1} c_{1} \int \psi_{j} d \xi \\
0.5 m l c_{2} \dot{\beta}_{2}^{2}+\sum_{j=1}^{r} m \dot{\eta}_{2 j} \dot{\beta}_{2} c_{2} \int \psi_{j} d \xi \\
0.5 m l c_{3} \dot{\beta}_{3}^{2}+\sum_{j=1}^{r} m \dot{\eta}_{3 j} \dot{\beta}_{3} c_{3} \int \psi_{j} d \xi
\end{array}\right] \in R^{3} \quad V_{2}=m\left[\begin{array}{c}
\sum_{j=1}^{r} \dot{\eta}_{1 j} \dot{\rho}_{1} c_{1} \int \psi_{j} d \xi \\
\sum_{j=1}^{r} \dot{\eta}_{2 j} \dot{\rho}_{2} c_{2} \int \psi_{j} d \xi \\
\sum_{j=1}^{r} \dot{\eta}_{3 j} \dot{\rho}_{3} c_{3} \int \psi_{j} d \xi
\end{array}\right] \in R^{3} \\
& V_{4}=-m\left[\begin{array}{c}
c_{1} \dot{\rho}_{1} \dot{\beta}_{1} \int \psi_{1} d \xi \\
\vdots \\
c_{1} \dot{\rho}_{1} \dot{\beta}_{1} \int \psi_{r} d \xi \\
c_{2} \dot{\rho}_{2} \dot{\beta}_{2} \int \psi_{1} d \xi \\
\vdots \\
c_{2} \dot{\rho}_{2} \dot{\beta}_{2} \int \psi_{r} d \xi \\
c_{3} \dot{\rho}_{3} \dot{\beta}_{3} \int \psi_{1} d \xi \\
\vdots \\
c_{3} \dot{\rho}_{3} \dot{\beta}_{3} \int \psi_{r} d \xi
\end{array}\right] \in R^{3 r}
\end{aligned}
$$

where $: s_{i}:=\sin \left(\alpha_{i}-\beta_{i}\right)$ and $c_{i}:=\cos \left(\alpha_{i}-\beta_{i}\right)$

$$
\begin{gathered}
J_{\Gamma 1}=\left[\begin{array}{cccccc}
\cos \alpha_{1} & \sin \alpha_{1} & 0 & 0 & 0 & 0 \\
0 & 0 & \cos \alpha_{2} & \sin \alpha_{2} & 0 & 0 \\
0 & 0 & 0 & 0 & \cos \alpha_{3} & \sin \alpha_{3}
\end{array}\right] \in R^{3 \times 6} \\
J_{\Gamma 2}=\left[\begin{array}{cccccc}
s 2_{1} & c 2_{1} & 0 & 0 & 0 & 0 \\
0 & 0 & s 2_{2} & c 2_{2} & 0 & 0 \\
0 & 0 & 0 & 0 & s 2_{3} & c 2_{3}
\end{array}\right] \in R^{3 \times 6}
\end{gathered}
$$

where: $s 2_{i}:=-l \sin \beta_{i}-\cos \beta_{i} \sum_{j=1}^{r} \eta_{i j}$ and $c 2_{i}:=l \cos \beta_{i}-\sin \beta_{i} \sum_{j=1}^{r} \eta_{i j}$ 


$$
J_{\Gamma 3}=\left[\begin{array}{cccccc}
-1 & 0 & -1 & 0 & -1 & 0 \\
0 & -1 & 0 & -1 & 0 & -1 \\
s 3_{1} & -c 3_{1} & s 3_{2} & -c 3_{2} & s 3_{3} & -c 3_{3}
\end{array}\right] \in R^{3 \times 6}
$$

where: $s 3_{i}:=e_{i x}, c 3_{i}:=e_{i y}$

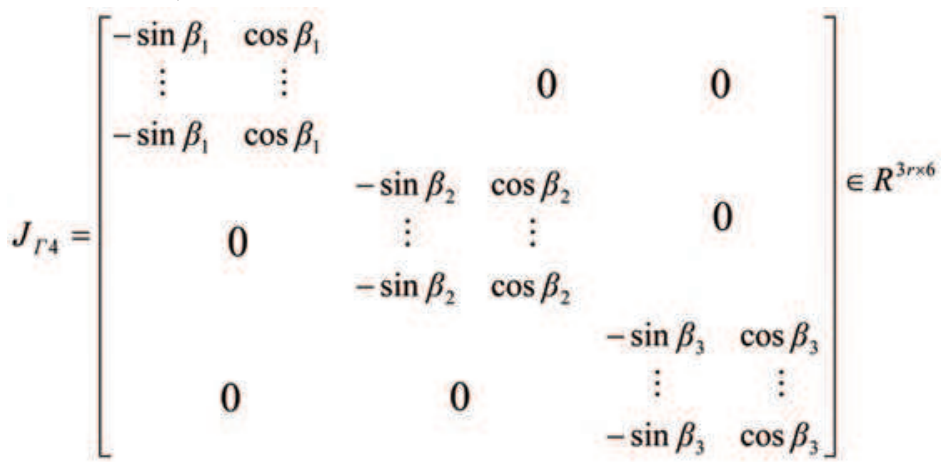

\section{Active vibration control}

If the intermediate linkages of the planar parallel manipulator are very stiff, an appropriate rigid body model based controller, such as a computed torque controller (Craig, 2003), can yield good trajectory tracking of the manipulator. However, structural flexibility of the linkages transfers unwanted vibration to the platform, and may even lead to instability of the whole system. Since control of linear motions of the sliders alone can not result in both precise tracking of the platform and vibration attenuation of the linkages simultaneously, an additional active damping method is proposed through the use of smart material. As discussed, the vibration damping controller proposed here is applied separately to a PVDF layer and PZT segments, and the performance of each actuator is then compared. Attached to the surface of the linkage, both of these piezoelectric materials generate shear force under applied control voltages, opposing shear stresses which arise due to elastic deformation of the linkages.

The integrated control system for the planar parallel manipulator proposed here consists of two components. The first component is a proportional and derivative (PD) feedback control scheme for the rigid body tracking of the platform as given below:

$$
u_{i}(t)=-k_{P}\left(\rho_{d i}-\rho_{i}\right)-k_{d}\left(\dot{\rho}_{d i}-\dot{\rho}_{i}\right), \quad i=1,2,3
$$

where $k_{p}$ and $k_{d}$ are a proportional and a derivative feedback gain respectively. $\rho_{d i}$ and $\dot{\rho}_{d i}$ are desired displacement and velocity of the $i^{\text {th }}$ slider respectively. This signal is used as an input to electrical motors actuating ball-screw mechanisms for sliding motions. In the following, we introduce the second component of the integrated control system separately, for each of the piezoelectric materials examined, a PVDF layer and PZT segments, shown respectively in Fig. 7 and 8. 


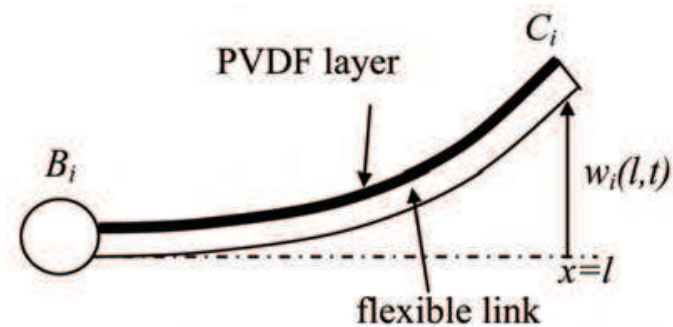

Fig. 7. Intermediate link with PVDF layer

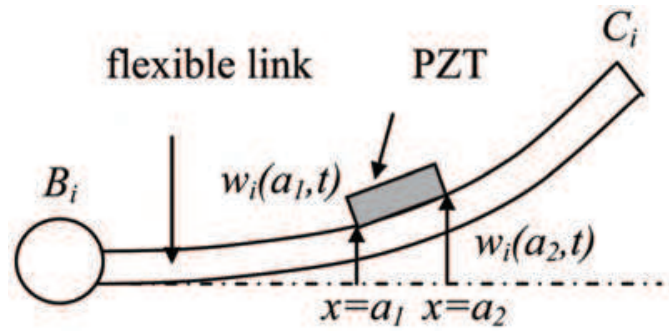

Fig. 8. Intermediate link with PZT actuator

\subsection{PVDF actuator control formulation}

A PVDF layer can be bonded uniformly on the one side of the linkages of the planar parallel manipulator, as shown in Fig. 7. When a control voltage, $v_{i}$, is applied to the PVDF layer, the virtual work done by the PVDF layer is expressed as

$$
\delta W_{P V D F}=c v_{i}(t) \sum_{j=1}^{r} \psi_{j}^{\prime}(l) \delta \eta_{i j}
$$

where $c$ is a constant representing the bending moment per volt (Bailey \& Hubbard, 1985) and $l$ is the link length. (.) implies differentiation with respect to $x$. If the control voltage applied to the PVDF layer, $v_{i}$, is formulated as

$$
v_{i}(t)=-k_{I} \dot{w}_{i}^{\prime}(l, t) \quad i=1,2,3
$$

the slope velocity of the linkages, $\dot{w}^{\prime}(l, t)$, converges to zero, assuming no exogenous disturbances applied to the manipulator, hence vibration of the linkages is damped out. Since the slope velocity, $\dot{w}^{\prime}(l, t)$, is not easily measured or estimated by a conventional sensor system, an alternative scheme, referred to as the L-type method (Sun \& Mills, 1999), is proposed as follows:

$$
v_{i}(t)=-k_{I} \dot{w}_{i}(l, t) i=1,2,3
$$

Instead of the slope velocity, $\dot{w}^{\prime}(l, t)$, the linear velocity, $\dot{w}(l, t)$, is employed in formulation of the control law. The linear velocity, $\dot{w}(l, t)$, can be calculated through the integration of 
the linear acceleration measured by an accelerometer installed at the distal end of the linkages, $C_{i}$. The shape function, $\psi_{j}(\xi=1)$, and its derivative, $\psi_{j}^{\prime}(\xi=1)$, have same trend of variation at the distal end of the linkages, $C_{i}$, in all vibration modes, as shown in Fig. 6 ;

$$
\psi_{j}(\xi=1) \psi_{j}^{\prime}(\xi=1) \geq 0 j=1,2,---, r
$$

Therefore, the control system maintains stability when employing the L-type method to formulate the control voltages, $v_{i}$.

\subsection{PZT actuator control formulation}

PZT actuators are manufactured in relatively small sizes, hence several PZT segments can be bonded together to a flexible linkage to damp unwanted vibrations. Assuming that only one PZT segment is attached to each intermediate linkage of the planar parallel manipulator, as shown in Fig. 8, the virtual work done by the PZT actuator is expressed as

$$
\delta W_{P Z T}=c v_{i}(t) \sum_{j=1}^{r}\left[\psi_{j}^{\prime}\left(a_{2}\right)-\psi_{j}^{\prime}\left(a_{1}\right)\right] \delta \eta_{i j}
$$

$a_{1}$ and $a_{2}$ denote the positions of the two ends of the PZT actuator measured from $B_{i}$ along the intermediate linkage, as shown in Fig. 8. As the PVDF layer is, the PZT actuator is controlled using the L-type method as

$$
v_{i}(t)=-k_{I}\left[\dot{w}_{i}\left(a_{2}, t\right)-\dot{w}_{i}\left(a_{1}, t\right)\right] i=1,2,3
$$

In contrast to the PVDF layer bonded uniformly to the manipulator linkages, the performance of the L-type scheme for the PZT actuator depends on the location of the PZT actuator. In order to achieve stable control performance, the PZT actuator should be placed in a region along the length of the linkage i.e. $x \in\left[a_{1}, a_{2}\right]$ as discussed in (Sun \& Mills, 1999), where $\psi_{j}(x)$ and $\psi_{j}^{\prime}(x)$ have the same trend of variation,

$$
\left(\psi_{j}\left(a_{2}\right)-\psi_{j}\left(a_{1}\right)\right)\left(\psi_{j}^{\prime}\left(a_{2}\right)-\psi_{j}^{\prime}\left(a_{1}\right)\right) \geq 0
$$

As the number of vibration modes increases, it is difficult to satisfy the stability condition, given in equation(50), for higher vibration modes, since the physical length of a PZT actuator is not sufficiently small.

\section{Simulation results}

Simulations are performed to investigate vibrations of the planar parallel manipulator linkages and damping performance of both piezoelectric actuators used in the manipulator with structurally-flexible linkages. Specifications of the manipulator for simulations are listed in Table 1. The first three modes are considered in the dynamic model, i.e. $r=3$. A sinusoidal function with smooth acceleration and deceleration is chosen as the desired input trajectory of the platform;

$$
x_{P}=\frac{x_{f}}{t_{f}} t-\frac{x_{f}}{2 \pi} \sin \left(\frac{2 \pi}{t_{f}} t\right)
$$


Considering the target-performance in an electrical assembly process, such as wire bonding in integrated circuit fabrication, the goal for the platform is designed to move linearly $2 \mathrm{~mm}$ $\left(x_{f}\right)$ within $10 \mathrm{msec}\left(t_{f}\right)$. Feedback gains of the control system for the slider actuators are listed in Table 2. The feedback gain for piezoelectric actuators, $k_{I}$, is selected so that the control voltage, applied to the PVDF layer, does not exceed 600 Volts. A fourth order Runge-Kutta method was used to integrate the ordinary differential equations, given by Equation (42) at a control update rate of $1 \mathrm{msec}$, using MATLAB ${ }^{\mathrm{TM}}$ software. Parameters of piezoelectric materials, currently manufactured, are listed in Table 3 . The placement position of the PZT actuator is adjusted to $a_{1}=0.66, a_{2}=0.91$, so that the first two vibration modes satisfy the stability condition given in equation (50).

Results of the PVDF layer are shown in Figures 9-12. Figure 9 shows that the error profile of the manipulator platform exhibits large oscillation at the initial acceleration, but continuously decreases due to the damping effect of the PVDF layer applied to the flexible linkages. The error profile of the platform without either of PVDF or PZT, labeled as "no damping" in Figure 9, shows typical characteristics of an undamped system. With Figure 10 showing deformation of the linkages on $C_{i}$, it reveals that the PVDF layer can damp structural vibration of the linkages in a gradual way. The first three vibration modes are illustrated in Figure 11. The first mode has twenty times the amplitude than the second mode, and one hundred times the amplitude than the third mode. The control output for the first slider actuator is shown in the upper plot of Figure 12, and control voltage for the first PVDF layer is shown in the lower plot of Figure 12. The control voltage, applied to the PVDF layer, decreases as the amplitude of vibration does.

Results of the PZT actuator are shown in Figures 13-17. Comparing Figure 13 with Figure 9, the PZT actuator exhibits better damping performance than the PVDF layer. The error profile of the platform, with the PZT actuator activated, enters steady state quickly and does not exhibit any vibration in steady state. The structural vibrations of the linkages, illustrated in Figure 14, are completely damped after $60 \mathrm{msec}$. The first three vibration modes are shown in Figure 15. The first mode has ten times the amplitudes than the other modes. Since the PZT actuator has higher strain constant than the PVDF, the PZT actuator can generate large shear force with relatively small voltage applied. The maximum voltage of the lower plot of Figure 16 is about 200 Volts, while that of the Figure 12 reaches 600 Volts. Due to the length of the linkage and the PZT actuator applied to the linkage, only the first two modes satisfy the stability condition, given by equation (50). However, this has little effect on damping performance, as shown in Figure 14 since the first two modes play dominant roles in vibration. If the placement of the PZT actuator change to $a_{1}=0.4, a_{2}=0.65$, only the first mode satisfies the stability condition, which leads to divergence of vibration modes, as shown in Figure 17.

\section{Conclusion}

In this chapter, the equations of motion for the planar parallel manipulator are formulated by applying the Lagrangian equation of the first type. Introducing Lagrangian multipliers simplifies the complexities due to multiple closed loop chains of the parallel mechanism and the structurally flexible linkages. An active damping approach applied to two different piezoelectric materials, which are used as actuators to damp unwanted vibrations of flexible 
linkages of a planar parallel manipulator. The proposed control is applied to PVDF layer and PZT segments. An integrated control system, consisting of a PD feedback controller, applied to electrical motors for rigid body motion control of the manipulator platform, and a L-type controller applied to piezoelectric actuators to damp unwanted linkage vibrations, is developed to permit the manipulator platform to follow a given trajectory while damping vibration of the manipulator linkages. With an L-type control scheme determining a control voltage applied, the piezoelectric materials have been shown to provide good damping performance, and eventually reduce settling time of the platform of the planar parallel manipulator. Simulation results show that the planar parallel manipulator, with the lightweight linkages, during rigid body motion, undergoes persistent vibration due to high acceleration and deceleration. Additionally, the PZT actuator yields better performance in vibration attenuation than the PVDF layer, but may enter an unstable state if the position of the PZT actuator on the linkage violates the stability condition for the dominant vibration modes. In the near future, we will perform vibration experiments with a prototype planar parallel manipulator based on presented simulation results.

\begin{tabular}{ccc}
\hline Platform & $\begin{array}{c}\text { side length } \\
\text { mass }\end{array}$ & $0.1 \mathrm{~m}$ \\
& $0.2 \mathrm{~kg}$ \\
\hline Slider & mass & $0.2 \mathrm{~kg}$ \\
\hline Linear guide (Ball- & stroke & $0.4 \mathrm{~m}$ \\
screw) & incline angle & $150 \circ, 270 \circ, 30^{\circ}$ \\
\hline \multirow{2}{*}{ Link } & length & $0.2 \mathrm{~m}$ \\
& density & $2770 \mathrm{~kg} / \mathrm{m}^{3}$ \\
& modulus & $73 \mathrm{GPa}$ \\
\hline
\end{tabular}

Table 1. Specification of the planar parallel manipulator

\begin{tabular}{cc}
\hline $\mathrm{k}_{\mathrm{p}}$ & $10,000 \mathrm{~N} / \mathrm{m}$ \\
$\mathrm{k}_{\mathrm{d}}$ & $500 \mathrm{~N}$-sec/m \\
& $4,000 \mathrm{~V}$-sec/m for PVDF \\
$\mathrm{k}_{\mathrm{I}}$ & $1,500 \mathrm{~V}$-sec/m for PZT
\end{tabular}

Table 2. Feedback control gains

\begin{tabular}{ccc}
\hline & PVDF & PZT \\
\hline modulus & $2 \mathrm{GPa}$ & $63 \mathrm{GPa}$ \\
length & $0.2 \mathrm{~m}$ & $0.05 \mathrm{~m}$ \\
thickness & $0.28 \mathrm{~mm}$ & $0.75 \mathrm{~mm}$ \\
width & $0.025 \mathrm{~m}$ & $0.025 \mathrm{~m}$ \\
density & $1800 \mathrm{~kg} / \mathrm{m}^{3}$ & $7600 \mathrm{~kg} / \mathrm{m}^{3}$ \\
$\mathrm{~d}_{31}$ & $22 * 10^{-12} \mathrm{~m} / \mathrm{V}$ & $110 * 10^{-12} \mathrm{~m} / \mathrm{V}$ \\
\hline
\end{tabular}

Table 3. Parameters of piezoelectric materials 


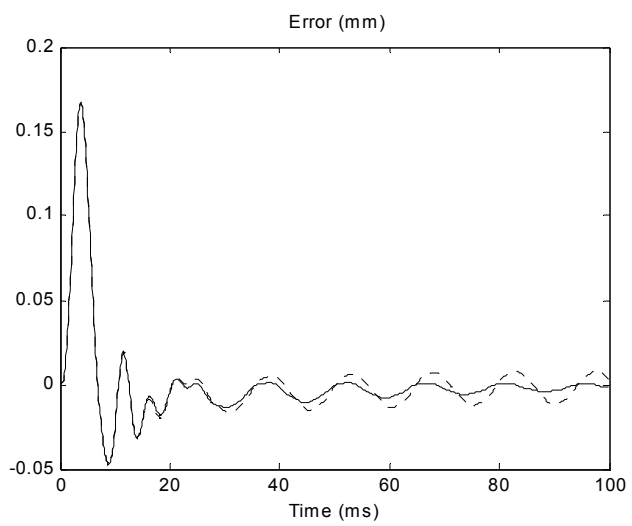

Fig. 9. Error profile of the platform (dotted: no damping, solid: with PVDF layer)
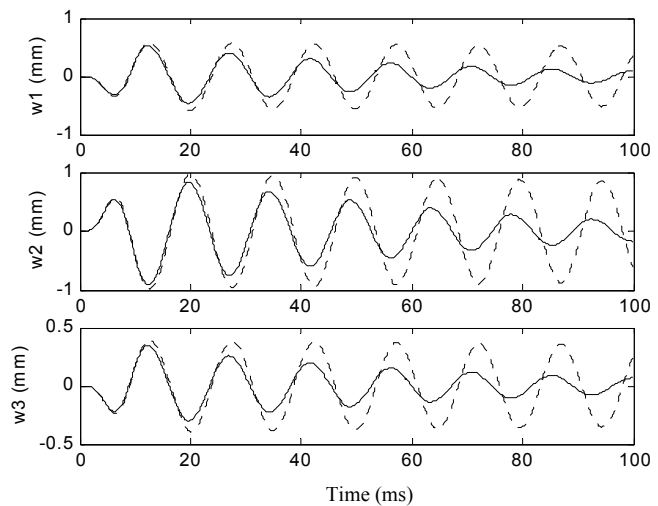

Fig. 10. Flexible deformation of each link (dotted: no damping, solid: with PVDF layer)
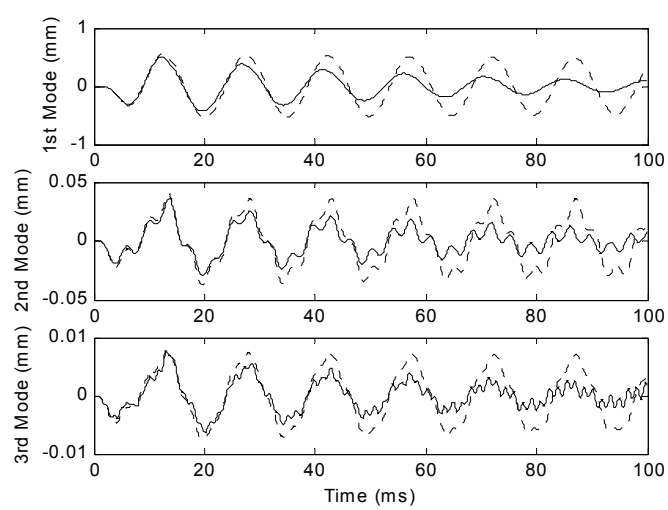

Fig. 11. The first three vibration modes of the first link (dotted: no damping, solid: with PVDF layer) 

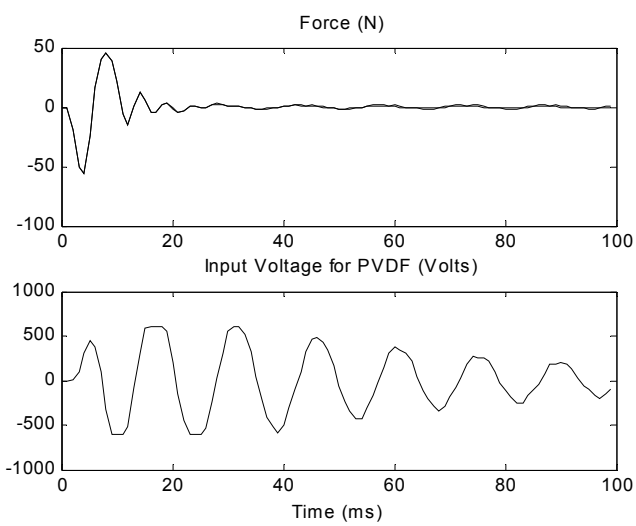

Fig. 12. Control output for the first link

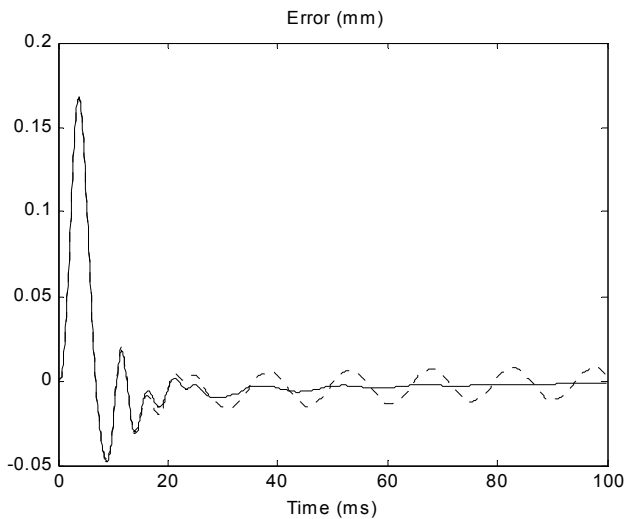

Fig. 13. Error profile of platform (dotted: no damping, solid: with PZT actuator)
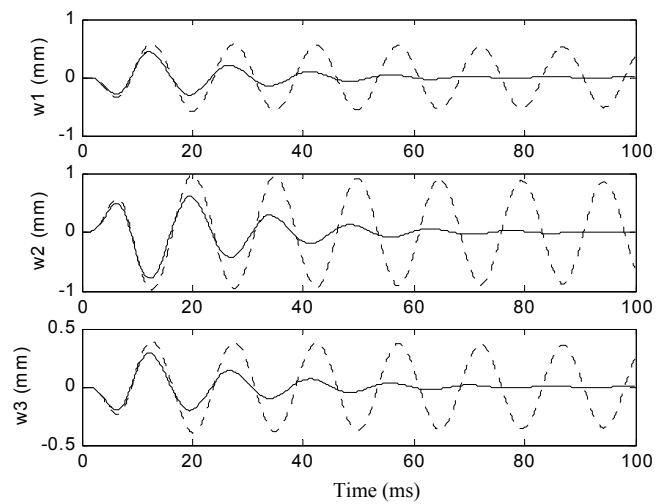

Fig. 14. Flexible deformation of each link (dotted: no damping, solid: with PZT actuator) 

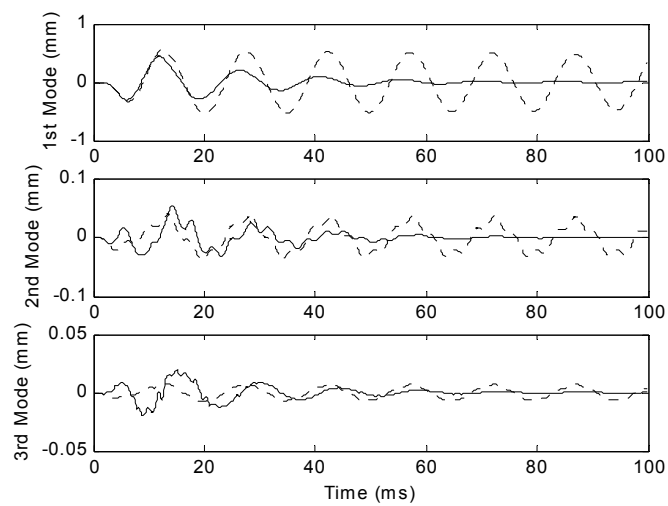

Fig. 15. The first three vibration modes of the first link(dotted: no damping, solid: with PZT actuator)
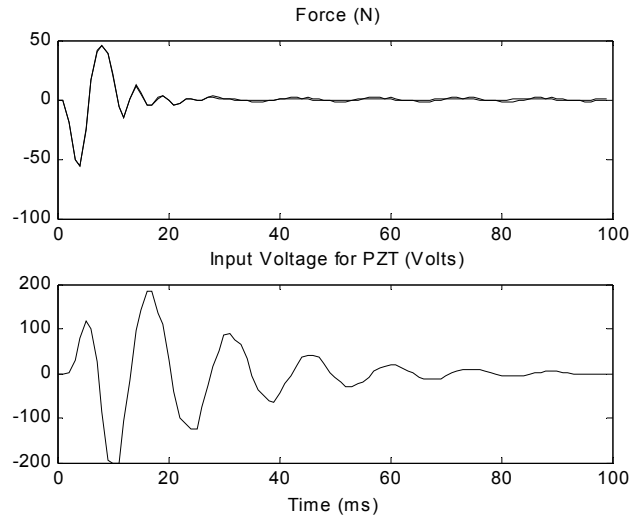

Fig. 16. Control output for the first link
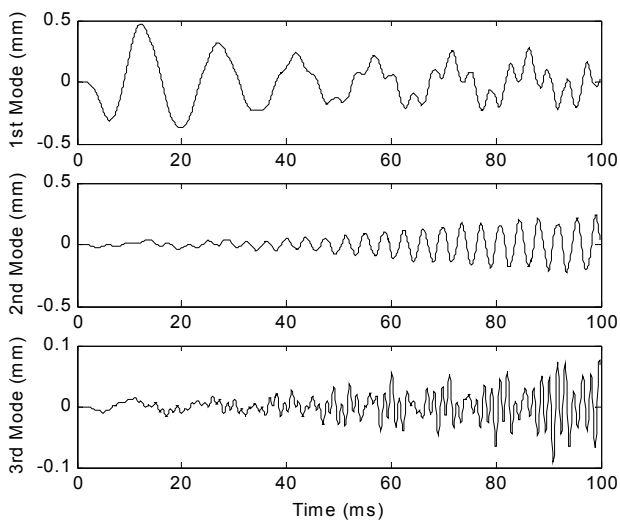

Fig. 17. The first three vibration modes of the first link with PZT actuator located on inappropriate place 


\section{References}

Arai, T.; Cleary, K., Homma, K., Adachi, H., \& Nakamura, T. (1991), Development of parallel link manipulator for underground excavation task, Proceedings of International Symposium on Advanced Robot Technology, pp. 541-548, Tokyo, Japan, March 1991

Bailey, T. \& Hubbard, J. (1985), Distributed piezoelectric-polymer active vibration control of a cantilever beam, Journal of Guidance, Control and Dynamics, Vol. 8, No. 5, pp. 605611, 0731-5090

Bellezza, F.; Lanari, L. \& Ulivi, G. (1990), Exact modeling of the flexible slewing link, Proceedings of IEEE International Conference on Robotics and Automation, pp. 734-739, 0-8186-9061-5, Cincinnati, USA, May 1990, IEEE

Craig, J. J. (2003), Introduction to Robotics, Prentice-Hall, ISBN-10: 0387985069

Fattah, A.; Angeles, J. \& Misra, K. A. (1995), Dynamic of a 3-DOF spatial parallel manipulator with flexible links, Proceedings of IEEE International Conference on Robotics and Automation, pp. 627-632, 0-7803-1965-6, Nagoya, Japan, May 1995, IEEE

Genta, G. (1993), Vibration of structures and machines, Springer, ISBN-10: 0387985069

Gosselin, C. \& Angeles, J. (1990), Singularity analysis of closed-loop kinematic chains, IEEE Transactions on Robotics and Automation, Vol. 6, No. 3, pp. 281-290, 1552-3098

Gosselin C.; Lemieux, S., \& Merlet, J.-P. (1996), A new architecture of planar three- degreeof-freedom parallel manipulator, Proceedings of IEEE International Conference on Robotics and Automation, pp. 3738-3743, 0-7803-2988, Minneapolis, USA, April 1996, IEEE

Heerah, I.; Kang, B., Mills, J. K., \& Benhabib, B. (2002), Architecture selection and singularity analysis of a 3-degree-of-freedom planar parallel manipulator, Proceedings of ASME 2002 Design Engineering Technical Conferences and Computers and Information in Engineering Conference, pp. 1-6, 791836037, Montreal, Canada, October 2002, ASME

Kang, B.; Yeung, B., and Mills, J. K. (2002), Two-time scale controller design for a high speed planar parallel manipulator with structural flexibility, Robotica, Vol. 20, No. 5, pp. 519-528, 0263-5747

Kozak, K.; Ebert-Uphoff, I., \& Singhose, W. E. (2004), Locally linearized dynamic analysis of parallel manipulators and application of input shaping to reduce vibrations, ASME Journal of Mechanical Design, Vol. 126, No. 1, pp. 156-168, 1050-0472

Low, K. H. \& Vidyasagar, M. (1988), A Lagrangian formulation of the dynamic model for flexible manipulator systems, ASME Journal of Dynamic Systems, Measurement, and Control, Vol. 110, pp. 175-181, 0022-0434

Merlet, J.-P. (1996), Direct kinematics of planar parallel manipulators, Proceedings of IEEE International Conference on Robotics and Automation, pp. 3744-3749, 0-7803-2988, Minneapolis, USA, April 1996, IEEE

Sun, D. \& Mills, J. K. (1999), PZT actuator placement for structural vibration damping of high speed manufacturing equipment, Proceedings of the American Control Conference, pp. 1107-1111, 0780349903, San Diego, USA, June 1999, IEEE 
Toyama, T.; Shibukawa, T., Hattori, K., Otubo, K., and Tsutsumi, M. (2001), Vibration analysis of parallel mechanism platform with tilting linear motion actuators, Journal of the Japan Society of Precision Engineering, Vol. 67, No. 9, pp. 458-1462, 0916$78 \mathrm{X}$ 


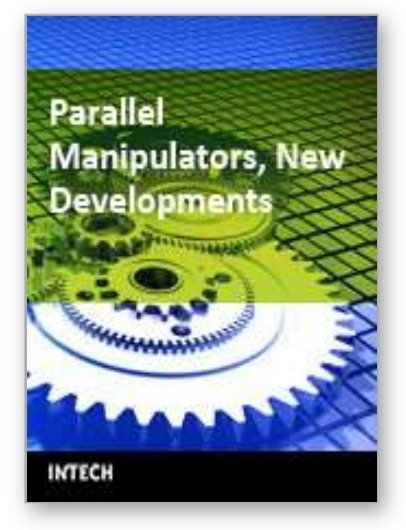

\author{
Parallel Manipulators, New Developments \\ Edited by Jee-Hwan Ryu
}

ISBN 978-3-902613-20-2

Hard cover, 498 pages

Publisher I-Tech Education and Publishing

Published online 01, April, 2008

Published in print edition April, 2008

Parallel manipulators are characterized as having closed-loop kinematic chains. Compared to serial manipulators, which have open-ended structure, parallel manipulators have many advantages in terms of accuracy, rigidity and ability to manipulate heavy loads. Therefore, they have been getting many attentions in astronomy to flight simulators and especially in machine-tool industries. The aim of this book is to provide an overview of the state-of-art, to present new ideas, original results and practical experiences in parallel manipulators. This book mainly introduces advanced kinematic and dynamic analysis methods and cutting edge control technologies for parallel manipulators. Even though this book only contains several samples of research activities on parallel manipulators, I believe this book can give an idea to the reader about what has been done in the field recently, and what kind of open problems are in this area.

\title{
How to reference
}

In order to correctly reference this scholarly work, feel free to copy and paste the following:

Bongsoo Kang and James K. Mills (2008). Dynamic Modelling and Vibration Control of a Planar Parallel Manipulator with Structurally Flexible Linkages, Parallel Manipulators, New Developments, Jee-Hwan Ryu (Ed.), ISBN: 978-3-902613-20-2, InTech, Available from:

http://www.intechopen.com/books/parallel_manipulators_new_developments/dynamic_modelling_and_vibratio n_control_of_a_planar_parallel_manipulator_with_structurally_flexible_

\section{INTECH}

open science | open minds

\author{
InTech Europe \\ University Campus STeP Ri \\ Slavka Krautzeka 83/A \\ 51000 Rijeka, Croatia \\ Phone: +385 (51) 770447 \\ Fax: +385 (51) 686166 \\ www.intechopen.com
}

\author{
InTech China \\ Unit 405, Office Block, Hotel Equatorial Shanghai \\ No.65, Yan An Road (West), Shanghai, 200040, China \\ 中国上海市延安西路65号上海国际贵都大饭店办公楼 405 单元 \\ Phone: +86-21-62489820 \\ Fax: +86-21-62489821
}


(C) 2008 The Author(s). Licensee IntechOpen. This chapter is distributed under the terms of the Creative Commons Attribution-NonCommercialShareAlike-3.0 License, which permits use, distribution and reproduction for non-commercial purposes, provided the original is properly cited and derivative works building on this content are distributed under the same license. 